\title{
Rapidité et durée d'action du paclobutrazol sur la croissance végétative et la fructification du pêcher en fonction du mode d'application du produit et de la concentration utilisée
}

\author{
Shao-Hua LI, Claude BUSSI (*) \& Pierre ATGER (*) \\ Département d'Horticulture, Université Agricole de Huazhong, Wuhan, République Populaire de Chine \\ (*) I.N.R.A.-S.R.I.V., Domaine de Gotheron, F 26320 St-Marcel-les-Valence
}

RÉSUMÉ

\begin{abstract}
Deux concentrations de paclobutrazol (PP333), 500 p.p.m. et 1000 p.p.m., et 2 modes d'application, traitement par pulvérisation foliaire et par absorption racinaire, ont été étudiés sur une variété très vigoureuse de pêcher Nectared 4 - en mesurant la cinétique de la croissance des pousses et des fruits, la longueur des entre-næeuds, laccroissement de la surface de section du tronc, le rendement, le poids moyen des fruits et la densité de floraison. Les résultats révèlent les principaux effets du paclobutrazol sur le pêcher : une réduction de la croissance des rameaux accompagnée d'un raccourcissement des entre-nœuds et d'une amélioration du calibre des fruits. La durée de l'effet du PP333 dépend du mode d'application et de la concentration utilisée. Par contre, la rapidité de manifestation de l'effet du produit varie seulement en fonction du mode d'application.

Une hypothèse est proposée concernant l'action du PP333 sur la croissance de la pèche en fonction de ses phases de développement :

Le PP333 freinerait les divisions cellulaires pendant la phase de multiplication cellulaire mais améliorerait le grandissement cellulaire au cours de la phase ultérieure de développement du fruit. Cette dernière action peut être interprétée comme une «stimulation » indirecte, par diminution de l'effet compétiteur des pousses.
\end{abstract}

Mots clés additionnels: Prunus persica (L.) Batsch; régulateur de croissance, PP333, croissance du végétal, développement des fruits. to concentration and application method.

Paclobutrazol (PP333) was applied to peach cv. Nectared 4 with 2 application methods (foliar spray and soil treatment) at 2 concentrations ( 500 p.p.m. and 1000 p.p.m.) and tested for its rapidity and duration of effect on growth and cropping. The effects of the product were studied on fruit and shoot growth patterns, internode length of shoot, increment of trunk sectional area, yicld, fruit size and flower bud density. Shoot growth reduction with shortened internodes and fruit size amelioration were observed following PP333 application. The duration of product effect depends on both application method and concentration. The rapidity of product action, however, was only related to application method. Furthermore, it was suggested that a double action of PP333 on fruit growth might occur according to the development phase of the fruit : PP333 may decrease fruit cell division during the first stage of fruit growth and improve fruit cell enlargement during the following stages. This last action may result from an indirect «stimulation», by reduction of shoot competition.

Additional key words : Prunus persica (L.) Batsch; growth regulator, PP333, vegetative growth, fruit growth.

\section{INTRODUCTION}

Actuellement, la tendance à des densités de plantation élevées pour le pêcher et le coût élevé de la taille (en particulier l'intervention en cours de végétation: taille en vert) justifient les nombreuses recherches sur le contrôle chimique du développement des arbres. 
Le paclobutrazol (PP333), un nouveau régulateur de croissance des plantes, semble présenter de l'intérêt dans ce sens pour les producteurs. En effet, de multiples travaux sur le pommier (JAMES, 1982; WILLIAMS \& EdGerton, 1983 ; Quinlan \& RichaRdSON, 1984), les agrumes (ARON et al., 1985), l'abricotier (ClANET \& SAlles, 1986) et le pêcher (EREZ, 1986) ont montré qu'il était très efficace pour limiter la croissance végétative des arbres fruitiers.

Ce produit peut également influencer les caractéristiques de la fructification, par exemple: induction ou initiation florale (CLANET \& SALLES, 1986), chute des jeunes fruits (LI, 1985; MARINI, 1986), et développement des fruits (CURRY \& WILlIAMS, 1983; EREZ, 1986; Greene, 1986).

Le paclobutrazol est absorbé au niveau des feuilles et des tiges des plantes. Il a été montré que ce produit pouvait aussi être fixé sur le complexe argilo-humique du sol et assimilé au niveau des racines (Aron et al., 1985), ce qui le distingue des autres régulateurs de croissance. La circulation du produit se réalise alors par la voie du xylème (DAlziel \& LAWrence, 1984). Ainsi, il apparaît particulièrement intéressant d'entreprendre une étude sur les modes d'application de PP333.

L'objectif de nos travaux est de contribuer à une meilleure connaissance de ce produit, en précisant sa rapidité d'action et la durée de son effet en fonction de 2 méthodes d'application : traitement au sol et traitement foliaire. Les observations porteront plus particulièrement sur la croissance végétative et la fructification.

\section{MATÉRIELS ET MÉTHODES}

Cette étude a été effectuée en 1985 et en 1986 sur le verger expérimental du domaine I.N.R.A.-S.R.I.V. de Gotheron à Valence. Le sol du verger se compose d'alluvions caillouteuses (30 à 50 p. 100 de cailloux) et sableuses (sur la fraction de terre fine: argile = 18 p. 100 , limon $=27$ p. 100 , sable $=54$ p. 100). La teneur en matière organique est de 1,3 p. 100 .

L'expérimentation a porté sur une seule variété très vigoureuse, Nectared 4, greffée sur GF 305. Les arbres plantés en 1982 y sont conduits en gobelet, à la distance de plantation de $2,5 \times 5 \mathrm{~m}$.

Les traitements au PP333 en 1985 et en 1986 peuvent être résumés de la façon suivante :

\begin{tabular}{|c|c|c|c|}
\hline \multirow{2}{*}{ Traitement } & \multicolumn{3}{|c|}{ Année } \\
\hline & 1985 & & 1986 \\
\hline 1 & Témoin & & Témoin \\
\hline 2 & 500 p.p.m. au sol & $20 \mathrm{j}+\mathrm{F} 2^{\mathrm{Z}}$ & aucun traitement \\
\hline 3 & 1000 p.p.m. au sol & $20 \mathrm{j}+\mathrm{F} 2$ & aucun traitement \\
\hline 4 & 500 p.p.m. foliaire & $24 \mathrm{j} .+F 2$ & aucun traitement \\
\hline 5 & 1000 p.p.m. foliairc & $24 \mathrm{j} .+\mathrm{F} 2$ & $\begin{array}{c}500 \text { p.p.m. foliaire } \\
24 \text { j. }+ \text { F2 }\end{array}$ \\
\hline
\end{tabular}

${ }^{\mathrm{L}} \mathrm{F} 2=$ pleine floraison.

En 1985, 2 concentrations du produit, 500 et 1000 p.p.m. de m.a. et 2 types d'applications, traite- ment au sol (sur la bande désherbée d'une largeur de $1 \mathrm{~m})$ et traitements foliaires ont été testés. Mais en 1986, seuls les arbres ayant reçu une application foliaire du produit à 1000 p.p.m. l'année précédente, ont été traités à nouveau à demi-concentration (500 p.p.m.) par pulvérisation foliaire.

Les traitements ont toujours été effectués à raison de 1000 litres de solution à l'hectare. Pour les traitements foliaires, le produit a été employé additionné d'un mouillant: l'agral 90 utilisé à 0,1 p. 100 . Tous les traitements au sol ont été effectués 20 jours après le stade pleine floraison (F2), et 24 jours après $F 2$ pour les traitements foliaires.

Le dispositif expérimental est constitué de 4 blocs. Dans chaque bloc, les traitements sont réalisés sur des parcelles élémentaires de 2 arbres.

Au cours de notre expérimentation, la croissance de 4 pousses vigoureuses de l'année par arbre, situées à la partie supérieure de la couronne, a été suivie à partir de la date de l'application du produit jusqu'à la fin de la saison. De plus, 8 rameaux mixtes sans anticipés ont été repérés en 1985 afin d'étudier l'effet du produit sur la croissance et sur la tenue des fruits. Cette dernière a été évaluée jusqu'à l'éclaircissage manuel effectué après la chute physiologique. L'accroissement relatif de la surface de section du tronc prise à une hauteur de $10 \mathrm{~cm}$ au-dessus du point de greffe est représenté par la valeur du rapport :

$\begin{array}{cc}\begin{array}{c}\text { Surface de section du tronc } \\ \text { à la fin de saison }\end{array} & \begin{array}{c}\text { Surface de section du tronc } \\ \text { au début de saison }\end{array} \\ \text { Surface de section du tronc au début de saison } & 100 \\ \text { p. } 100\end{array}$

La densité de floraison est déterminée par le nombre de bourgeons à fleurs/mètre d'après l'observation de 8 rameaux par arbres choisis au hasard après la chute des feuilles.

\section{RÉSULTATS}

\section{A. Cinétique de la croissance végétative de pousses}

En suivant l'allongement des pousses vigoureuses en 1985, nous avons constaté que l'effet du produit se manifeste plutôt par absorption foliaire que par assimilation racinaire (fig. 1). Pour les traitements foliaires (500 et 1000 p.p.m. de PP333), 14 jours après la pulvérisation, la vitesse de croissance des pousses a été significativement ralentie par rapport à celle du témoin, par contre cette différence entre le témoin et le traitement au sol n'est apparue que 30 jours après l'application du produit. Ensuite, les pousses de tous les arbres traités se sont développées plus lentement que celles des témoins, sauf pour le traitement 4 (500 p.p.m. sur feuilles). Pour ce dernier, l'effet retardant de croissance n'est plus significatif à partir du 19 juillet.

\section{B. Longueur des pousses et des entre-nœuds}

Le tableau 1 montre qu'une réduction très importante de la croissance des pousses a été obtenue en réponse 
TABLEAU

Effet du traitement au PP333 sur la croissance végétative du pêcher.

Effect of PP333 treatment on the vegetative growth of peach tree.

\begin{tabular}{|c|c|c|c|c|c|}
\hline Année & Traitement & $\begin{array}{l}\text { Longueur }(\mathrm{cm}) \\
\text { de pousses } \\
\text { vigoureuses } \\
\text { de l'année }\end{array}$ & $\begin{array}{l}\text { Nombre par rameau } \\
\text { de pousses de } \\
\text { l'année d'une longueur } \\
\text { supérieure à } 5 \mathrm{~cm}\end{array}$ & $\begin{array}{l}\text { Longueur des } \\
\text { entre-nouds }(\mathrm{cm})\end{array}$ & $\begin{array}{c}\text { Accroissement } \\
\text { relatif de la surface } \\
\text { de section du tronc } \\
(\%)\end{array}$ \\
\hline \multirow{6}{*}{1985} & 1 - témoin & $96,8 \mathrm{z}$ & 3,7 & 2,11 & 32,6 \\
\hline & 2 - 500 p.p.m. au sol & 66,5 & 3,5 & $1,51_{\mathrm{b}}$ & 8,4 \\
\hline & 3-1000 p.p.m. au sol & $64,0_{b}$ & 3,2 & $1,32_{\mathrm{b}}$ & $7,3_{\mathrm{b}}$ \\
\hline & 4 - 500 p.p.m. foliaire & $74,2^{\circ}$ & 4,8 & $1,52_{b}$ & $12,5^{\circ}$ \\
\hline & $5-1000$ p.p.m. foliaire & $59,8 \mathrm{~b}$ & 3,6 & $1,37_{\mathrm{b}}$ & $9,9 \mathrm{~b}$ \\
\hline & ppds à $5 \%$ & 15,1 & N.S. & 0,22 & 8,9 \\
\hline \multirow{6}{*}{1986} & 1 - témoin & $131,2 \mathrm{a}$ & - & 2,15 & $22,3_{b}$ \\
\hline & 2 - non traité & $92,7_{c}^{d}$ & - & $1,86_{b}$ & $16,9_{\mathrm{bc}}^{\circ}$ \\
\hline & 3 - non traité & $72,0_{d}$ & - & $1,63_{\mathrm{c}}$ & $11,5_{\mathrm{c}}$ \\
\hline & 4 - non traité & $114,6 \mathrm{~b}$ & - & 2,25 & 37,8 a \\
\hline & $5-500$ p.p.m. foliaire & $68,3_{\mathrm{d}}^{\circ}$ & - & $1,57_{\mathrm{c}}$ & $11,0_{c}^{\mathrm{a}}$ \\
\hline & ppds à $5 \%$ & 16,3 & - & 0,14 & 9,9 \\
\hline
\end{tabular}

$\mathrm{z}=$ Les valeurs suivies d'une même lettre ne sont pas significativement différentes.

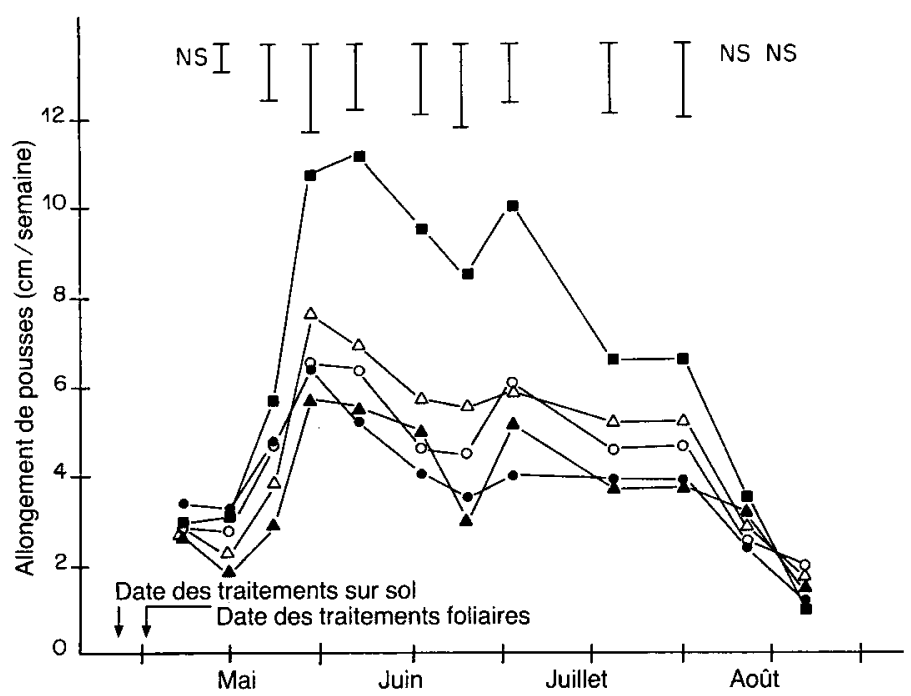

Figure 1

Allongement hebdomadaire des pousses en fonction du traitement au PP333 (1985) : témoin $\square-$ a, 500 p.p.m. au sol $\bigcirc-O$, 1000 p.p.m. au sol $\longrightarrow$ - 500 p.p.m. foliaire $\triangle \longrightarrow$, et 1000 p.p.m. foliaire $\mathbf{\Delta} \boldsymbol{\Lambda}$. Les barres verticales représentent la ppds à $5 \%$.

Weekly shoot increase related to PP333 treatments (1985): control $\square+\mathbf{\square}$, soil application at 500 p.p.m. $\bigcirc-0$, soil application at 1000 p.p.m., - - leaf application at 500 p.p.m. $\triangle \longrightarrow \triangle$ and leaf application at 1000 p.p.m. $\mathbf{-}$ - $\mathbf{- V e r t i -}$ cal bars represent L.S.D. at $5 \%$.

aux applications de paclobutrazol en 1985. Toutefois, nous ne remarquons pas de différences significatives entre les traitements. En 1986, l'effet du produit sur la croissance des pousses se manifeste différemment suivant les traitements : les traitements 3 (1 000 p.p.m. au sol en 1985) et 5 (1 000 p.p.m. sur feuilles en 1985 et 500 p.p.m. sur feuilles en 1986) réduisent le plus fortement la croissance des pousses. Ensuite, le traitement 2 (500 p.p.m. sur sol en 1985) réduit plus la croissance que le traitement 4 (500 p.p.m. sur feuilles en 1985).
En réalité, cette réduction de la croissance de pousses s'est accompagnée d'une diminution de la longueur des entre-nœuds, mais pas de la réduction du nombre de pousses (tabl. 1). Une relation significative apparaît entre l'effet du traitement sur l'allongement des pousses et l'effet sur le raccourcissement des entre-nœuds (l'équation de la droite de régression s'établit ainsi avec les résultats des 2 années: $\mathrm{y}=0,622+0,0132 \mathrm{x}$ $r^{2}=0,87^{* *}$ y: longueur des entre-nœuds en $\mathrm{cm}$; $\mathrm{x}$ : longueur de pousse en $\mathrm{cm}$ ).

\section{TABLEAU 2}

Densité de floraison en fonction du traitement au PP333, exprimée en nombre de bourgeons à fleur par mètre de rameau.

Flower bud density according to PP333 treatments, expressed as number of flower buds per shoot meter.

\begin{tabular}{lcc}
\hline \hline & \multicolumn{2}{c}{ Année } \\
\cline { 2 - 3 } Traitement & 1985 & 1986 \\
\hline 1 & $80,1 \mathrm{c}^{\mathrm{z}}$ & $61,4 \mathrm{c}$ \\
2 & $120,6_{\mathrm{ab}}$ & $78,1 \mathrm{~b}_{\mathrm{b}}$ \\
3 & $136,4_{\mathrm{a}}$ & $87,4 \mathrm{a}$ \\
4 & $101,9 \mathrm{bc}$ & $55,3 \mathrm{c}$ \\
5 & $138,9_{\mathrm{a}}$ & $88,7 \mathrm{a}$ \\
\hline ppds à $5 \%$ & 31,2 & 8,8 \\
\hline Date d'observation & novembre 1985 & février 1987
\end{tabular}

$\mathrm{z}=$ Les valeurs suivies d'une même lettre ne sont pas significativement différentes.

\section{Accroissement relatif de la surface de section du tronc}

En 1985, les traitements au PP333 ont tous limité le grossissement du tronc comme le montrent les résultats du tableau 1. Mais en 1986, seuls les traitements 3 (forte dose de PP333 au sol en 1985) et 5 (traitement foliaire renouvelé en 1986) ont réduit la croissance du tronc. 


\section{Cinétique de la croissance des fruits}

Le rythme de croissance des fruits observé en 1985 sur tous les traitements tend à se distinguer de celui enregistré sur le témoin comme le montre la figure 2 .

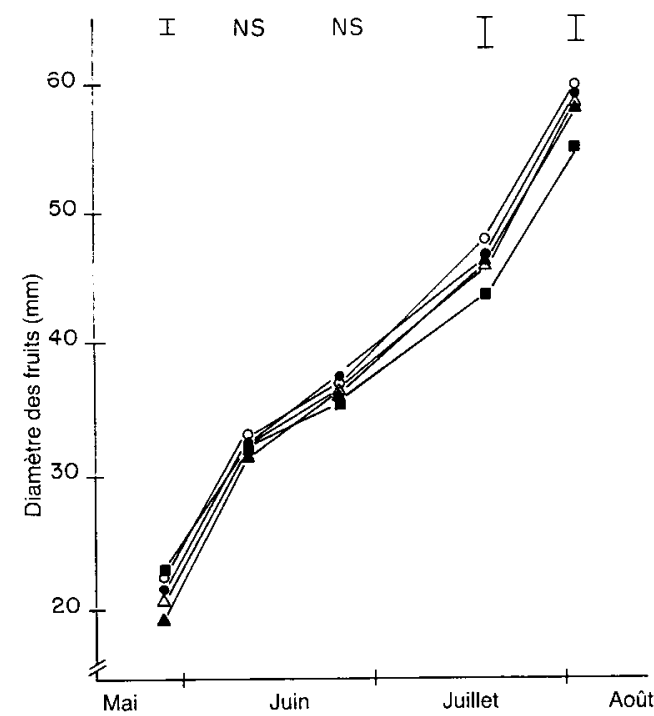

Figure 2

Rythme de croissance des fruits en fonction du traitement au PP333 (1985) : témoin $\mathbf{\square} \mathbf{\square}, 500$ p.p.m. au sol $\bigcirc-0,1000$ p.p.m. au sol —— 500 p.p.m. foliaire $\triangle \longrightarrow \triangle$, ef 1000 p.p.m. foliaire $\mathbf{\Delta}-\boldsymbol{\Delta}$. Les barres verticales représentent la ppds à $5 \%$. Fruit diameter increase in response to PP333 treatments (1985). control $\mathbf{\square}$ application at 1000 p.p.m. - leaf application at 500 p.p.m. $\triangle \longrightarrow \triangle$, and leaf application at 1000 p.p.m. $\mathbf{\Delta}-\mathbf{\Delta}$. Vertical bars represent L.S.D. at $5 \%$

On peut remarquer que pour tous les arbres traités au PP333, les fruits sont moins gros que ceux provenant des arbres témoins avant juin. Par la suite, les fruits sur les arbres traités ont grossi plus vite que ceux des arbres témoins. En conséquence, à partir de la mi-juillet, la situation est inversée : le calibre des fruits sur les arbres traités est plus important que celui des fruits provenant des arbres témoins.

\section{E. Densité de floraison}

Une application au PP333 augmente la densité de floraison pour tous les traitements au cours de ces 2 années (cf. tabl. 2) sauf en ce qui concerne le traitement 4 (500 p.p.m. foliaire en 1985). Pour ce dernier, l'effet obtenu a été très faible en 1985 et nul en 1986. De plus, la densité de floraison pour les traitements 3 et 5 est plus importante que celle obtenu pour le traitement 2 en 1986.

\section{F. Chute des fruits}

Une chute de fruits a été observée pour tous les traitements, mais elle ne se différencie pas de celle du témoin (résultats non présentés).

\section{G. Aspect économique}

Compte tenu de l'intérêt économique des traitements au PP333, nous avons rassemblé les résultats dans le tableau 3 , relatif à la production et au poids moyen des fruits pour ces 2 années, et au temps de taille seulement pour 1985.

Ces résultats sont les suivants :

- le traitement au PP333 n'a pas significativement influencé la production du pêcher pour les 2 années d'expérimentation ;

- le traitement au PP333 a amélioré le calibre des fruits par rapport au témoin en 1985 , mais cet effet ne persiste pas en 1986 ;

- au point de vue de la taille en vert, une réduction importante du temps de taille est obtenue grâce au

\begin{tabular}{|c|c|c|c|c|c|c|}
\hline & \multirow{2}{*}{ Traitement } & \multirow{2}{*}{$\begin{array}{l}\text { Production } \\
\text { (kg/arbre) }\end{array}$} & \multirow{2}{*}{$\begin{array}{l}\text { Poids moyen } \\
\text { des fruits } \\
\text { (g) }\end{array}$} & \multicolumn{3}{|c|}{ Temps de taille $(\mathrm{h} / \mathrm{ha})$} \\
\hline & & & & en vert & en hiver & total \\
\hline \multirow{6}{*}{1985} & 1 - témoin & 20,8 & $112,9 \mathrm{z}$ & 83,6 & 98,5 & 182,1 \\
\hline & 2- $\quad 500$ p.p.m. au sol & 22,1 & 130,4 & 44,7 & 83 & 127,7 \\
\hline & 3- 1000 p.p.m. au sol & 22,8 & $126,5_{\mathrm{ab}}^{\mathrm{a}}$ & 53,3 & 80,9 & 134,2 \\
\hline & 4 - 500 p.p.m. foliaire & 22,1 & $125, l_{a b}$ & 58,8 & 90,9 & 149,7 \\
\hline & $5-1000$ p.p.m. foliaire & 21,4 & $118,1_{b c}$ & 49,3 & 85,3 & 134,6 \\
\hline & ppds à $5 \%$ & NS & 8,92 & - & - & - \\
\hline \multirow{6}{*}{1986} & 1- témoin & 19,27 & 110,8 & - & - & - \\
\hline & 2 - non traité & 20,50 & 112,0 & - & 一 & - \\
\hline & 3 - non traité & 16,61 & 115,6 & - & - & - \\
\hline & 4 - non traité & 19,84 & 107,2 & - & - & - \\
\hline & 5 - 500 p.p.m. foliaire & 16,88 & 117,5 & - & - & 一 \\
\hline & ppds à $5 \%$ & NS & NS & - & - & - \\
\hline
\end{tabular}

z Les valeurs suivies d'une même lettre ne sont pas significativement différentes. 
traitement au paclobutrazol. Cette réduction est de l'ordre de 24,8 à 38,9 h/ha (soit 29,7 à 46,5 p. 100 de moins que les arbres témoins). Mais le gain de temps de taille d'hiver a été très faible.

\section{DISCUSSION}

Les essais effectués sur une variété très vigoureuse, Nectared 4 et portant sur divers traitements au paclobutrazol et différentes méthodes d'application ont mis en évidence une réduction très importante de la croissance du végétal (pousse, tronc) en $1^{\text {rc }}$ année consécutivement à l'application du produit. Cet effet est observable quelle que soit la concentration utilisée (500 p.p.m. et 1000 p.p.m. de m.a.), et quelle que soit la méthode d'application (au sol 20 jours après $F 2$ ou sur frondaison 24 jours après F2).

Toutefois, l'effet résiduel du produit en $2^{\mathfrak{c}}$ année varie :

\section{- En fonction de la méthode d'application.}

Pour la concentration de 500 p.p.m., le traitement au sol présente encore un effet l'année suivante. A cette même concentration, il ne se manifeste pas d'effet résiduel du traitement foliaire.

\section{- En fonction de la concentration.}

En traitement au sol, l'efficacité du paclobutrazol augmente de 500 p.p.m. à 1000 p.p.m.

D'autre part, le traitement au PP333 influence la fructification du pêcher.

Tout d'abord, la densité de floraison a été augmentée en $1^{\text {re }}$ année, consécutivement à l'application du PP333 et quelle que soit la technique de traitement, mais l'effet du traitement pour la $2^{\mathrm{e}}$ année dépend du mode d'application et de la concentration utilisée. Cette augmentation de la densité de floraison pourrait être essentiellement attribuable à un raccourcissement de la longueur des entre-nœuds des pousses. En effet, on observe une corrélation négative significative entre la longueur des entre-nœuds des arbres d'un traitement et leur densité de floraison (l'équation de la droite de régression s'écrit : $y=229,8-78,06 \times \mathrm{r}^{2}=0,82^{* *} ; \mathrm{y}=$ densité de floraison; $\mathrm{x}$ : longueur des entre-nœuds en $\mathrm{cm}$ ).

Le grossissement des fruits a été influencé pendant la première phase de leur croissance. Ceux-ci, sur les arbres traités, se développent d'abord moins vite que ceux des arbres témoins. Par contre, au cours de la phase finale de croissance, le phénomène s'inverse. Les mêmes résultats ont été obtenus en 1985 sur la même variété, dans une autre expérimentation avec le paclobutrazol (LI, 1985).

Ce phénomène peut suggérer les hypothèses suivantes :

1) Le PP333 freinerait d'abord le grossissement des fruits en début de croissance. Cette phase correspond à la période de division cellulaire qui se poursuivrait (JACKSON, 1968) jusqu'à environ 50 jours après le stade pleine floraison (soit jusqu'à fin-mai dans notre expérimentation).

Le principal mode d'action du PP333 consiste en une inhibition de la biosynthèse des gibbérellines dans les plantes (COUTURE, 1982). JACQMARD (1968), LIU \& LOY (1976) ont mis en évidence 2 actions de l'acide gibbérellique favorisant la prolifération cellulaire. D'abord, l'acide gibbérellique peut stimuler les cellules qui se trouvent à la phase $\mathrm{Gl}$ entre la mitose et la synthèse de l'ADN afin que celles-ci entrent dans la phase suivante, celle de la synthèse de l'ADN. En second lieu, l'acide gibbérellique peut raccourcir la durée de la phase de synthèse de l'ADN. Il y a tout lieu de penser que le bas niveau de cette hormone dans les fruits sur les arbres ayant reçu le traitement au PP333 ralentirait le processus de la division cellulaire. Il en résulterait une réduction du nombre de cellules des fruits sur ces arbres.

2) Le PP333 améliorerait la croissance finale des fruits, phase qui correspond à la période de grandissement cellulaire (JACKSON, 1968). Ce phénomène pourrait s'expliquer par l'amélioration de la situation nutritive pour les fruits des arbres traités:

L'effet du produit sur la croissance du végétal affaiblit la concurrence qui existe au niveau nutritionnel entre les pousses et les fruits, donnant l'avantage à ces derniers.

- Sur pommier, le PP333 augmente la teneur en chlorophylle des feuilles (STEFFENs et al., 1983). Dans ce cas, l'amélioration de l'assimilation chlorophyllienne augmenterait la production des hydrates de carbone par les feuilles, favorisant la nutrition et le grandissement des fruits.

- Sur pommier, le PP333 inhibe la respiration des tissus (SWIETLIK \& Miller, 1983), ce qui contribue à diminuer les pertes en produits résultant de la photosynthèse.

Ainsi, le calibre final du fruit à la récolte sur les arbres traités au PP333 dépend de l'équilibre entre les 2 actions précédemment décrites. Ceci explique peut-être la grande variabilité de l'influence du traitement au PP333 sur le calibre des fruits à la récolte, qui se distingue de celui observé sur la végétation, dans notre expérimentation et dans les travaux rapportés : soit une amélioration du calibre des fruits (LI, 1985; EREZ, 1986), soit une diminution du calibre des fruits (CuRRY \& Williams, 1983; GreENE, 1986), soit encore une absence d'effet apparent (MARINI, 1986).

\section{CONCLUSION}

Les travaux que nous avons effectués sur l'influence du traitement au paclobutrazol sur la végétation et la fructification de pêchers Nectared 4 en 1985 et en 1986 , nous permettent de préciser la rapidité et la durée de l'action du produit selon la concentration et le mode d'application.

- Au cours de l'année du traitement, nous avons constaté avec une concentration de 500 à 1000 p.p.m., la rapidité d'effet du produit en application foliaire par rapport à celle qui est obtenue après application au sol. Mais la concentration n'intervient pas à ce niveau, quelle que soit la méthode d'application utilisée.

- Par contre, la durée de l'effet résiduel du produit dépend de la méthode aussi bien que de la concentration d'application: l'effet du traitement au sol dure plus longtemps que celui du traitement foliaire. De même, l'effet obtenu à la concentration de 1000 p.p.m. est plus durable qu'à la concentration de 500 p.p.m.

Par ailleurs, cette étude confirme qu'une concentration de 500 à 1000 p.p.m. de paclobutrazol avec une dose de 1000 //ha est suffisante pour régulariser la 
croissance végétative du pêcher. Du point de vue de l'efficacité du produit, il semble que le paclobutrazol appliqué au sol présenterait plus d'intérêt comparativement à des pulvérisations sur frondaison. Cependant l'augmentation de la densité de floraison après application du paclobutrazol pourrait accentuer dans ces conditions le problème de l'éclaircissage des arbres.

\section{REMERCIEMENTS}

Nous remercions P. SARTRE pour sa collaboration efficace au niveau de la réalisation des essais sur le terrain, P. Rousset (Société SOPRA) pour la fourniture du produit, J. HuGARD (I.N.R.A.-Montpellier) et R. MONET (I.N.R.A.-Bordeaux) pour leurs judicieux conseils.

Nous ajoutons aussi tous nos remerciements aux lecteurs, J.L. REgnaRd (E.N.S.H.-Versailles) et H. Clanet (I.N.R.A.Montpellier) pour toutes les corrections apportées et suggestions émises pour améliorer ce travail.

\section{RÉFÉRENCES BIBLIOGRAPHIQUES}

Aron Y., Monselise S. P., Goren R., Costo J., 1985. Chemical control of vegetative growth in citrus trees by paclobutrazol. HortScience, 20 , 96-98.

Clanet H., Salles J. C., 1986. Contrôle de la croissance et de la fructification de l'abricotier par l'utilisation du paclobutrazol. Acta Hort., 160, 207-215.

Couture R. M., 1982. PP333: A new experimental plant growth regulator from ICI. Proc. Plant Growth Reg. Soc. Amer., 9, 59 p.

Curry E. A., Williams M. W., 1983. Promalin or GA3 increase pedicel and fruit length and leaf size of "Delicious" apples treated with paclobutrazol. HortScience, 18, 214-215.

Dalziel J., Lawrence D. K., 1984. Biochemical and biological effects of kaurene oxidase inhibitors, such as paclobutrazol. Brit. plant. Growth Regulat. Group. Monogr., 11, 43-57.

Erez A., 1986. Growth control with paclobutrazol of peaches growth in a meadow orchard system. Acta. Hort., 160, 217-225.

Greene D. W., 1986. Effect of paclobutrazol and analogs on growth, yield, fruit quality, and storage potential of "Delicious» apple. $J$. Amer. Soc. Hort. Sci., 111 , 328-332.

Jackson D. I., 1968. Gibberellin and the growth of peach and apricot fruits. Aust, J. biol. Sci., 21, 209-215.

Jacqmard A., 1968. Early effects of gibberellic acid on mitotic activity and DNA synthesis in the apical bud of Rudbeckia bicolor. Physiol. vég., $6,409-416$.
James D. Q., 1982. Recent developments and future prospects for the chemical control of tree growth. Compact. Fruit Tree, 15, 33-39.

Li S. H., 1985. Contribution à l'étude de l'éclaircissage chimique du pêcher (Prunus persica (L.) Batsch.). Mémoire de D.A.A. à l'E.N.S.A.Montpellier.

Liu P. B. W., Loy J. B., 1976. Action of gibberellic acid on cell proliferation in the subapical shoot meristem of watermelon seedlings. Amer. J. Bot., 63, 700-704.

Marini R. P., 1986. Growth and cropping of "Redhaven » peach trees following foliar application of flurprimidol and paclobutrazol. $J$. Amer. Soc. Hort. Sci., 111, 849-853.

Quinlan J. D., Richardson P. J., 1984. Effet of paclobutrazol (PP333) on apple shoot growth. Acta Hort., 146, 105-111.

Steffens G. L., Wang S. Y., Steffens C. L., Brennan T., 1983. Influence of paclobutrazol (PP333) on apple seedling growth and physiology. Proc. Plant Growth Reg. Soc. Amer., 10, 195-206.

Swietlik D., Miller S. S., 1983. The effect of paclobutrazol on growth and response to water stress of apple seedlings. J. Amer. Soc. Hort. Sci., 108, 1076-1080.

Williams M. W., Edgerton L. J., 1983. Vegetative growth control of apple and pear trees with ICI PP333 (paclobutrazol) a chemical analog of bayleton. Acta Hort., 137, 111-116. 